\title{
Bargaining, Coalitions and Externalities: A Comment on Maskin*
}

\author{
Geoffroy de Clippel ${ }^{\dagger}$ and Roberto Serrano
}

June 5, 2008

\begin{abstract}
We first observe that two of Maskin's results do not extend beyond three players: we construct a four-player partition function with nonpositive externalities whose unique solution is inefficient, as well as a four-player characteristic function that has a unique efficient solution for each ordering of the players, but for which the payoff vector obtained by averaging these solutions over the different orderings does not coincide with the Shapley value. On the other hand, we reinforce Maskin's insight that externalities may play a crucial role in generating inefficiency. Many existing solutions on how to share profits assume or derive the property of efficiency. Yet we argue that players may have an interest to choose with whom to bargain. We illustrate how this may trigger inefficiency, especially in the presence of externalities, even if bargaining among any group of agents results in an efficient distribution of the surplus they can produce. We also provide some sufficient conditions for efficiency.
\end{abstract}

JEL classification numbers: C7, D62

Keywords: externalities, coalition formation, Shapley value

\section{Introduction}

Eric Maskin devoted his Presidential Address to the Econometric Society (Maskin (2003)) to the study of cooperation in the presence of externalities. He considers the model of a partition function, a simple extension of the characteristic function where the profit of a coalition may vary with the coalitions that form in the complement (Thrall and Lucas (1963)). Inspired by a specific class of bargaining procedures, one for each possible ordering of the bargainers, Maskin introduces a new solution that determines the coalitions that form, and how the surplus is shared among their members.

\footnotetext{
* Serrano gratefully acknowledges the hospitality of Universidad Carlos III and CEMFI in Madrid, and the research support from Fundación Banco Herrero and Universidad Carlos III.

${ }^{\dagger}$ Department of Economics, Brown University; geoffroy_declippel@brown.edu

$\ddagger$ Department of Economics, Brown University and IMDEA-Ciencias Sociales; roberto_serrano@brown.edu
} 
Two main insights (obtained after proving existence and providing a characterization of an appealing set of equilibria) delivered by Maskin's procedure are as follows: ${ }^{1}$

- (1) The grand coalition forms if externalities are nonpositive, while inefficient outcomes may prevail if externalities are positive.

- (2) The average of the players' payoffs over all the possible orderings coincides with the Shapley value when externalities are absent.

The second part of Insight (1) is surprising, to the extent that in the cooperative approach to game theory, efficiency has been often assumed as an axiom for superadditive situations. Insight (2), on the other hand, speaks to the "legitimacy" of the first, in the sense that it is obtained with a procedure that would still yield one of the classic solutions in the no-externality domain.

Maskin proves that these results hold when restricting attention to three-player partition functions. In Section 2, we begin by pointing out that, for more than three players, additional assumptions beyond superadditivity are required to obtain these results in Maskin's bargaining procedure. Specifically, Example 1 considers a four-player partition function with nonpositive externalities for which the grand coalition does not form in its unique Maskin solution (for some ordering of the players), and Example 2 describes another four-player game without externalities for which the grand coalition forms in its unique solution for each ordering of the players, but the average of the payoffs is not the Shapley value.

Nevertheless, Section 3 in this paper makes the case that insights (1) and (2) efficiency when externalities are "small" relative to the grand coalition surplus and inefficiencies under positive externalities, in models that yield the Shapley value with no external effects- can be obtained in many settings. To do so, instead of relying on a particular bargaining procedure, we turn to a more general approach. This generality stems from two considerations:

- (i) in the coalition formation stage, one can use a plethora of solution concepts (such as dominant coalition structures, the core, or the subgame perfect equilibria of a class of non-cooperative sequential procedures), and

- (ii) when a coalition structure is formed, the payoffs to players can be determined by a large class of solutions, as long as they are consistent with axioms characterizing the Shapley value of games without externalities. These axioms include efficiency, anonymity and some form of marginalism, as explored in de Clippel and Serrano (2008), thereby covering all solutions identified through axiomatic extensions of the Shapley value; see the references of that paper for a detailed list.

That externalities may be a source of inefficiency seems at odds with many alternative models that have been designed to better understand cooperation in these environments.

\footnotetext{
${ }^{1}$ Maskin (2003) restricts attention to superadditive partition functions, in which the surplus created by the grand coalition exceeds the sum of those created by any partition thereof. We shall do the same here.
} 
From a non-cooperative point of view, the various procedures studied by Macho-Stadler et al. (2006) and Hafalir (2007) all lead to an efficient outcome whether externalities are present or not. A straightforward application of Hart and Mas-Colell's (1996) or Pérez-Castrillo and Wettstein's (2001) arguments to partition functions also leads to the formation of the grand coalition. From an axiomatic point of view, division rules, also called values, are required to be efficient in most, if not all, existing works. Indeed, why consider the possibility of leaving some profit undistributed when designing a value?

The idea we develop in Section 3 is that, even if profits are shared efficiently within each coalition, inefficiency may occur because players may find it in their best interest to restrict the set of players with whom to bargain. Thus, we shall study a two-stage game. Players decide with whom to negotiate in a first stage. Bargaining on how to share the profit takes place in a second stage, as soon as a coalition structure has cristalized. Driven by a concern of deriving robust qualitative results, we will consider a large class of solutions that encompass both non-cooperative and axiomatic ideas (as in items (i) and (ii) above).

Our findings are as follows:

- (a) The presence of externalities may be a robust cause of inefficiency when players strategize to decide with whom to negotiate. But other culprits for inefficiencies exist: in general, the "Coase theorem logic" does not necessarily apply in our framework because the players' hands are tied by the bargaining procedure or the axioms behind the value when deciding with whom to bargain.

- (b) By construction, when no externalities are considered, our predictions agree with the Shapley value if the grand coalition forms, or with the Shapley value of each relevant subgame otherwise.

- (c) We provide sufficient conditions on the partition function under which the grand coalition is efficient and the unique dominant partition. A dominant partition, when it exists, provides a very robust prediction of the coalitions that should form, pretty much independent of the solution concept employed in the coalition formation stage.

In conclusion, Maskin's Insights (1) and (2) are compatible with numerous scenarios of coalition formation and externalities.

\section{Some Limits to Maskin's General Results}

Maskin's solution is inspired by a specific bargaining protocol. Cooperation builds up in many steps as bargaining unfolds. Given an exogenous ordering, the first player offers to the next one a monetary compensation in order to become his representative in further negotiations. If the second player accepts, then the first player will proceed in the same way with the third player. If, on the other hand, the second player refuses, then the first two players will compete for the representation of the third player. Competition here means that both players will simultaneously make a monetary offer to the third player 
for his cooperation. That third player will then have the option to either accept one of the two offers, or none at all. The bargaining game goes on in this way, buying out the cooperation of players through the competition of coalitional leaders, until the last player in the ordering receives some offer and decides which one to accept, if any. The coalitional leaders then choose collective actions so as to maximize profits (given the other coalitions formed), and fulfill the contracts they signed by paying the players that agreed to subordinate their actions. Assuming that the environment is quasilinear, there is no loss of generality to restrict attention to partition functions to summarize the coalitional profit anticipated by each coalition, as a function of the coalition structure that forms, instead of describing explicitly the strategic options available to each coalition, and their consequences. Maskin's solution to the problem of cooperation in partition functions coincides with a subset of the subgame perfect equilibrium outcomes of the bargaining procedure just described: a specific equilibrium of the competition game between coalitional leaders is selected, whose outcome is that of a second-price auction played in dominant strategies. It should be easy to follow the computations hereafter without introducing further notations to define Maskin's solution more formally. The interested reader is referred to Maskin (2003) for details.

Maskin (2003, Theorem 2) states that his solution is efficient (the grand coalition forms) if externalities are non-positive. The proof is given under the assumption that there are only three players. The next example implies that additional assumptions are required to obtain this result in Maskin's procedure if there are more than three players.

Example 1 Consider the following four-player characteristic function: ${ }^{2} v(\{i\})=0$ for each player $i, v(\{a, c\})=1, v(\{a, d\})=10, v(\{c, d\})=30, v(\{a, c, d\})=31, v(\{a, b\})=$ $v(\{b, d\})=40, v(\{a, b, d\})=41, v(\{b, c\})=100, v(\{b, c, d\})=v(\{a, b, c\})=101$, $v(\{a, b, c, d\})=120$. Notice that the game is (strictly) superadditive. Consider the natural ordering $[a b c d]$. There is a subgame perfect equilibrium (satisfying Maskin's refinement) with player d joining a for a price of 1 , and player $c$ joining $b$ for free. The final payoff is $(9,100,0,1)$ and is inefficient. We check the equilibrium conditions starting from the last subgames. Consider the competition between a and coalition $\{b, c\}$ for player $d$. Player $b$ does not want to outbid player a, because the contribution of player $d$ to coalition $\{b, c\}$ equals 1. Player a does not want to lower his bid because he gets a null payoff without the cooperation of player $d$. Consider now players a and $b$ competing for $c$. If player a proposes a strictly positive bid, then $b$ will win player $d$ in the next step, as $v(\{b, d\})-v(\{b\})=40>30=v(\{a, c, d\})-v(\{a, c\})$. Player a ends up with a payoff lower than 1, which is worse than 9, his equilibrium payoff. We move backwards to the first stage. We know that player b gets 100 if he refuses the offer from a (this is on the equilibrium path). Is player a better off by proposing a higher payoff to player b? Suppose that player a gets player $b$. If player c refuses an offer from player a in the next round, then they will compete for player d. Player $c$ will win player $d$ for a price of 1 , as $v(\{c, d\})-v(\{c\})=30>1=v(\{a, b, d\})-v(\{a, b\})$. Hence, player a must offer a

\footnotetext{
${ }^{2}$ Inefficiency would prevail, even if the example was slightly modified so as to have strictly negative externalities.
} 
compensation of at least 29 to player $c$ if he wants to attract him. Otherwise, player a stays with $b$, and $c$ goes with $d$. Player a gets a negative payoff in both cases.

Maskin (2003, Theorem 3) states that the average of the players' payoffs over the different orderings coincides with the Shapley value in the absence of externalities. Again, the proof assumes that there are only three players. One may be tempted to think that the result follows from the fact that Maskin's payoff vectors would coincide with the marginal contribution vectors, as the Shapley value is known to coincide with the average of the marginal contribution vectors. Actually this first intuition is false, as can be checked by computing the marginal contribution vectors in Maskin (2003, Example 1). Yet, it turns out, quite surprisingly, that the sum of Maskin's payoff vectors over the two orderings where $i$ is last coincides with the sum of the two marginal contribution vectors over these same orderings, hence providing an alternative proof of Maskin's result for three-player characteristic functions. But this coincidence does not extend beyond three players. We already know from our first example that Maskin (2003, Theorem 3) cannot hold in general with more than three players, since the average of his payoff vectors is inefficient in that example. One could still hope that the result holds provided that the average is efficient. The following example implies that further assumptions are needed.

Example 2 Consider the following characteristic function: $v(\{a, b, c, d\})=10, v(\{a, b, c\})=$ $9, v(\{a, b, d\})=8, v(\{a, c, d\})=7, v(\{b, c, d\})=6, v(\{a, b\})=v(\{a, c\})=v(\{a, d\})=1$ and $v(S)=0$ for any other coalition $S$. Let's compute Maskin's and the marginal contribution vectors.

\begin{tabular}{|c|c|c|}
\hline$o$ & $M A(o)$ & $M C(o)$ \\
\hline$[a b c d]$ & $(10,0,0,0)$ & $(4,6,0,0)$ \\
{$[a c b d]$} & $(10,0,0,0)$ & $(4,0,6,0)$ \\
{$[b a c d]$} & $(1,9,0,0)$ & $(7,3,0,0)$ \\
{$[b c a d]$} & $(0,10,0,0)$ & $(1,3,6,0)$ \\
{$[c a b d]$} & $(2,0,8,0)$ & $(8,0,2,0)$ \\
{$[c b a d]$} & $(0,1,9,0)$ & $(1,7,2,0)$ \\
\hline
\end{tabular}

\begin{tabular}{|c|c|c|}
\hline$o$ & $M A(o)$ & $M C(o)$ \\
\hline$[b c d a]$ & $(0,10,0,0)$ & $(0,3,6,1)$ \\
{$[b d c a]$} & $(0,10,0,0)$ & $(0,3,1,6)$ \\
{$[c b d a]$} & $(0,1,9,0)$ & $(0,7,2,1)$ \\
{$[c d b a]$} & $(0,0,10,0)$ & $(0,1,2,7)$ \\
{$[d b c a]$} & $(0,2,0,8)$ & $(0,8,1,1)$ \\
{$[d c b a]$} & $(0,0,1,9)$ & $(0,1,8,1)$ \\
\hline
\end{tabular}

\begin{tabular}{|c|c|c|}
\hline$o$ & $M A(o)$ & $M C(o)$ \\
\hline$[a c d b]$ & $(10,0,0,0)$ & $(4,0,6,0)$ \\
{$[a d c b]$} & $(10,0,0,0)$ & $(4,0,0,6)$ \\
{$[c a d b]$} & $(2,0,8,0)$ & $(8,0,2,0)$ \\
{$[c d a b]$} & $(0,0,10,0)$ & $(1,0,2,7)$ \\
{$[d a c b]$} & $(3,0,0,7)$ & $(9,0,0,1)$ \\
{$[d c a b]$} & $(0,0,1,9)$ & $(1,0,8,1)$ \\
\hline
\end{tabular}

\begin{tabular}{|c|c|c|}
\hline$o$ & $M A(o)$ & $M C(o)$ \\
\hline$[a b d c]$ & $(10,0,0,0)$ & $(4,6,0,0)$ \\
{$[a d b c]$} & $(10,0,0,0)$ & $(4,0,0,6)$ \\
{$[b a d c]$} & $(1,9,0,0)$ & $(7,3,0,0)$ \\
{$[b d a c]$} & $(0,10,0,0)$ & $(1,3,0,6)$ \\
{$[d a b c]$} & $(3,0,0,7)$ & $(9,0,0,1)$ \\
{$[d b a c]$} & $(0,2,0,8)$ & $(1,8,0,1)$ \\
\hline
\end{tabular}

The sum of the $M A$ vectors equals $(72,64,56,48)$, while the sum of the $M C$ vectors equals $(78,62,54,46)$. These sums are different because $v(\{a, b\}), v(\{a, c\})$ and $v(\{a, d\})$ are relatively small and therefore do not influence the computation of the $M A$ vector. Actually, the vectors in the middle column coincide with the MA vectors of the game $v^{\prime}$ where 
$v^{\prime}(\{a, b\})=v^{\prime}(\{a, c\})=v^{\prime}(\{a, d\})=0$ and $v^{\prime}(S)=v(S)$ for all the other coalitions $S$. Their mean coincides with the Shapley value of the game $v^{\prime}$. The Shapley value, on the other hand, is sensitive to the modification of the worth of coalitions $\{a, b\},\{a, c\}$ and $\{a, d\}$. The power of player $a$ is increased and therefore he gets two additional units of surplus from each of the other players.

\section{Choosing with Whom to Negotiate}

We study a two-stage cooperation process. Players first choose with whom they want to negotiate. The outcome of this first stage is a coalition structure $\Pi$. Payoffs are then determined within each atom of $\Pi$ in the second stage by applying a value. The process can be solved by backward induction. To this end, we next discuss both values for a given coalitional structure, and solutions to the coalition formation problem.

\subsection{Payoff Distribution for a Given Coalition Structure}

Suppose that a coalition structure $\Pi$, not necessarily the grand coalition, has materialized, meaning that players within each atom $S$ of $\Pi$ have agreed to negotiate with each other, and with no agents from other atoms. For coalition $S \in \Pi$, we thus have to restrict attention to the partition function $v_{(S, \Pi)}$ derived from $v$ as follows: $v_{(S, \Pi)}\left(S^{\prime}, \Pi^{\prime}\right):=$ $v\left(S^{\prime}, \Pi^{\prime} \cup \Pi_{-S}\right)$, for each embedded coalition $\left(S^{\prime}, \Pi^{\prime}\right)$ defined over $S$. A plethora of models can be applied to figure out the payoff distribution for each $S \in \Pi$. Indeed, a host of linear and even non-linear values that have been proposed satisfy the properties of efficiency, anonimity and weak marginality, as defined in de Clippel and Serrano (2008). It follows from our previous work that they all coincide on the class of partition functions with symmetric externalities. ${ }^{3}$ All these values are also monotonic, again in the sense of de Clippel and Serrano (2008). This implies that they all fall within some common bounds that emerge from the resolution of a linear programming problem, even if they do not all coincide when externalities are not symmetric.

Formally, an $(S, \Pi)$-value is a function $\sigma^{(S, \Pi)}$ that assigns to every partition function $v$ (defined for the grand coalition $N)$ a unique utility vector $\sigma^{(S, \Pi)}(v) \in \mathbb{R}^{S}$. It is then straightforward to phrase the properties of $(S, \Pi)$-Anonymity, $(S, \Pi)$-Efficiency, Weak $(S, \Pi)$-Marginality, and $(S, \Pi)$-Monotonicity, as in de Clippel and Serrano (2008), simply by thinking of $S$ as a grand coalition on its own, whose members are taking $\Pi_{-S}$ as given.

For instance, $(S, \Pi)$-Efficiency would require that $\sum_{i \in S} \sigma_{i}^{(S, \Pi)}(v)=v(S, \Pi)$. The details are left to the interested reader. Let $S h^{S}$ denote the Shapley value for characteristic functions defined over $S$ :

$$
\operatorname{Sh}_{i}^{S}(v):=\sum_{T \subseteq S \text { s.t. }} \frac{(t-1) !(s-t) !}{s !}\left[v(T)-v\left(T_{-i}\right)\right]
$$

\footnotetext{
${ }^{3}$ Externalities that do not depend on players' names, but only on the cardinality of the sets of players affected.
} 
for each player $i \in S$, and each characteristic function $v$ defined over $S$. We can then rephrase the first two propositions of de Clippel and Serrano (2008) as follows:

Proposition 1 Let $\sigma^{(S, \Pi)}$ be an $(S, \Pi)$-value that satisfies $(S, \Pi)$-Anonymity, $(S, \Pi)$ Efficiency, and Weak $(S, \Pi)$-Marginality. Let $u$ be a symmetric partition function, and let $v$ be a characteristic function. Then $\sigma_{i}^{(S, \Pi)}(u+v)=\frac{u(S, \Pi)}{s}+S h_{i}^{S}\left(v_{S}\right)$, where $v_{S}$ is the reduced characteristic function defined over $S$ by $v_{S}(T)=v(T)$, for each $T \subseteq S$.

Proposition 2 If $\sigma^{(S, \Pi)}$ is an $(S, \Pi)$-value that satisfies $(S, \Pi)$-Anonymity, $(S, \Pi)$-Efficiency, and $(S, \Pi)$-Monotonicity, then one can solve a linear programming problem as in de Clippel and Serrano (2008, Section 4) so as to find two real numbers $\mu_{i}^{(S, \Pi)}(v)$ and $\nu_{i}^{(S, \Pi)}(v)$ so that

$$
\sigma_{i}^{(S, \Pi)}(v) \in\left[\mu_{i}^{(S, \Pi)}(v), \nu_{i}^{(S, \Pi)}(v)\right]
$$

for each $i \in S$.

\subsection{Solutions to the Problem of Coalition Formation}

Our next task is to suggest how coalition structures form in the first place. Let $x$ be the function that summarizes the outcome of the second stage of the process, as broadly discussed in the previous subsection. It is thus a function that associates a vector in $\mathbb{R}^{N}$ to each coalition structure. With our approach, we fix a list $\left(\sigma^{(S, \Pi)}\right)$ of values, and $x_{i}(\Pi)=\sigma_{i}^{(S, \Pi)}(v)$, for each coalition structure $\Pi$, and each player $i$, with $S$ being the atom of $\Pi$ to which $i$ belongs.

We focus now on the question of coalition formation. One advantage of our approach is the relative simplicity of this problem, because the players' final payoffs are given as a function of the coalitions that form. ${ }^{4}$ Still, different scenarios may lead to different outcomes. We articulate our arguments around three solutions.

(i) The first solution is extremely appealing, when it exists. Coalition $S$ is strictly dominant if each member of $S$ ranks $S$ as the best coalition, whatever the other players do, i.e.,

$$
x_{i}(\Pi)>x_{i}\left(\Pi^{\prime}\right)
$$

for each $i \in S$, each partition $\Pi$ that contains $S$, and each partition $\Pi^{\prime}$ that does not contain $S$. If $S$ is strictly dominant, we can be confident that it will form in frictionless scenarios of coalition formation. ${ }^{5}$ Assuming that $S$ forms, there may now be another coalition that is strictly dominant for the remaining players (i.e., in $N \backslash S$ ). Continuing in this fashion may lead to a unique partition. A partition $\Pi$ is strictly dominant if it

\footnotetext{
${ }^{4}$ In the absence of externalities, i.e., when $x_{i}(\Pi)=x_{i}\left(\Pi^{\prime}\right)$ for each $i$ and each $\Pi$ and $\Pi^{\prime}$ such that the atoms to which $i$ belongs are the same in both partitions, these problems of coalition formation have been referred to as "hedonic games." Thus, our problem could be referred to as an hedonic game with externalities.

${ }^{5}$ This impression is confirmed by our two other solutions. If a coalition structure $\Pi$ belongs to our second or third solution, and $S$ is strictly dominant, then $S \in \Pi$.
} 
is obtained by the iterative formation of strictly dominant coalitions. Clearly, a strictly dominant partition often fails to exist, but if there is one such partition, then it is unique.

(ii) The core is our second solution to the coalition formation stage. A coalition $S$ blocks a partition $\Pi^{\prime}$ if $x_{i}(\Pi)>x_{i}\left(\Pi^{\prime}\right)$ for each $i \in S$ and each partition $\Pi$ that contains $S$. The core is the set of partitions that are not blocked by any coalition. Instead of trying to determine the partition that will form, we eliminate those that are unstable. We want to eliminate with confidence and this is why we require the members of the objecting coalitions to be better off whatever the other players do after the deviation. ${ }^{6}$ Any other expectation when considering the formation of an objecting coalition would lead to a smaller core. If there is a strictly dominant partition, then this is the only partition in the core. When there is no such partition, then the core may be empty (Shenoy (1979, example 7.5)) or may contain more than one partition.

(iii) The third solution we suggest is based on a specific non-cooperative scenario of coalition formation. Fix an order $\pi$ for the players in $N$. Following the order $\pi$, each player $i \in N$ announces a coalition $S$ that contains $i$. The outcome of this sequential move game of perfect information is a coalition structure, in which a coalition $S$ forms if and only if each player in $S$ has announced the coalition $S$. If there is a strictly dominant partition, then this is the equilibrium outcome for every order of the players. The proposed coalition formation game is a finite horizon extensive form of perfect information. It admits at least one subgame perfect equilibrium ${ }^{7}$ and it is "almost always" unique. ${ }^{8}$ On the other hand, the equilibrium outcome may depend on the ordering of the players.

\subsection{Inefficiency and its Causes}

We are now ready to draw some qualitative conclusions about the outcomes of our twostage cooperation process. Our first observation is that inefficiency may be entirely due to the presence of externalities in some situations.

Example 3 This example features prominently in Maskin (2003). A similar example was first proposed by Ray and Vohra (1999, Example 1.2). It describes a simple "free rider" problem created by a public good that can be produced by each two-player coalition. The set of agents is $N=\{a, b, c\}$, and the partition function is: $v(N)=24$; $v(\{a, b\})=12 ; v(\{a, c\})=13 ; v(\{b, c\})=14 ; v(\{i\},\{\{i\},\{j, k\}\})=9$ for all $i, j, k ;$ $v(\{i\},\{\{i\},\{j\},\{k\}\})=0$ for all $i, j, k$.

Suppose that the value $\sigma^{(S, \Pi)}$ satisfy $(S, \Pi)$-Anonymity, $(S, \Pi)$-Efficiency and Weak $(S, \Pi)$-Marginality, for each embedded coalition $(S, \Pi)$. Externalities are symmetric in $v$ and the analysis presented in Subsection 3.1 thus implies that the payoffs in each coalition structure are: $x(\{N\})=(7.5,8,8.5) ; x(\{a\},\{b, c\})=(9,7,7) ; x(\{b\},\{a, c\})=$ $(6.5,9,6.5) ; x(\{c\},\{a, b\})=(6,6,9) ; x(\{a\},\{b\},\{c\})=(0,0,0)$.

\footnotetext{
${ }^{6}$ Such a construction is reminiscent of the maximin (or $\alpha-$ ) representations of games in strategic form.

${ }^{7}$ Hence the subgame perfect equilibrium outcomes associated to this class of bargaining procedures do not necessarily belong to the core (as the core may be empty).

${ }^{8}$ If each player's payoffs at the terminal nodes are different, then there exists a unique subgame perfect equilibrium, which yields the backwards induction outcome.
} 
There is no strictly dominant partition. The core of the coalition formation problem is

$$
\{\{N\},\{\{a\},\{b, c\}\},\{\{b\},\{a, c\}\},\{\{c\},\{a, b\}\}\} .
$$

Apart from each of the three partitions containing a two-player coalition and the free rider as a singleton, the grand coalition is also stable because of our very conservative definition of blocking: an individual player does not block because he is afraid of the "incredible threat" posed by the two-player coalition, i.e., that they will not cooperate after he leaves. Our third solution takes care of this. Whatever the order $\pi$, the coalition structure predicted by the unique subgame perfect equilibrium has the first mover alone in his singleton coalition, rationally anticipating that after he chooses to be alone, the other two will join together in the two-player coalition, as will surely happen. Of course, the specific coalition structure that emerges from the sequential game depends on the order, which assigns different bargaining power to players as a function of how early they speak in the game: each of the partitions containing a two-player coalition is the subgame perfect equilibrium outcome of the game for a given ordering. On the other hand, $v$ is superadditive, and hence the efficient coalition structure is the grand coalition. The outcome of our two-stage cooperation process admits inefficient partitions (and in the case of the sequential game, it consists exclusively of inefficient partitions).

Consider now the modified partition function $v^{\prime}$ where the only change with respect to $v$ is that the agents do not benefit from positive externalities:

$$
v^{\prime}(\{i\},\{\{i\},\{j, k\}\})=v^{\prime}(\{i\},\{\{i\},\{j\},\{k\}\})=0 \text { for all } i, j, k .
$$

This is a characteristic function. Hence the outcomes obtained by applying the list of values $\left(\sigma^{(S, \Pi)}\right)$ must coincide with the Shapley value of the reduced characteristic functions. We have: $x^{\prime}(\{N\})=(7.5,8,8.5) ; x^{\prime}(\{a\},\{b, c\})=(0,7,7) ; x^{\prime}(\{b\},\{a, c\})=(6.5,0,6.5)$; $x^{\prime}(\{c\},\{a, b\})=(6,6,0) ; x^{\prime}(\{a\},\{b\},\{c\})=(0,0,0)$.

The efficient coalition structure, $\{N\}$, is now strictly dominant and is thus expected to be the outcome of most scenarios of coalition formation in the first stage. We conclude that inefficiency may be entirely attributed to the presence of externalities in some situations.

But externalities are not the only cause of inefficiency:

Example 4 Let $N=\{a, b, c\}$ be the set of agents and let $v$ be the following characteristic function: $v(N)=18 ; v(\{a, b\})=16 ; v(\{a, c\})=14 ; v(\{b, c\})=12 ; v(\{i\})=0$ for all $i$. The outcomes obtained by applying a list of values $\left(\sigma^{(S, \Pi)}\right)$ that satisfy $(S, \Pi)$-Anonymity,

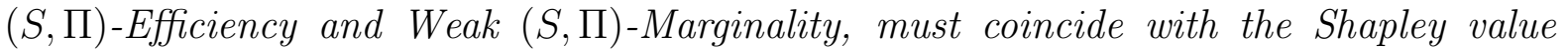
of the reduced characteristic functions. We have: $x(\{N\})=(7,6,5) ; x(\{a\},\{b, c\})=$ $(0,6,6) ; x(\{b\},\{a, c\})=(7,0,7) ; x(\{c\},\{a, b\})=(8,8,0) ; x(\{a\},\{b\},\{c\})=(0,0,0)$.

The coalition structure $\{\{a, b\},\{c\}\}$ is strictly dominant (first eliminate $\{a, b\}$ and then $\{c\})$. Hence it is also the unique core partition and it is the outcome of our third solution, for the six possible orderings of the players. Yet $v$ is superadditive and hence the grand coalition is the only efficient outcome. 
We close this subsection by offering one more example that illustrates the relevance of externalities in determining the outcome of the cooperation process, and its efficiency. Indeed, we will see that the application of distinct values that differ only in the way that externalities are taken into account to determine payoffs may lead to very different predictions in terms of the coalitions that form in the first stage.

Example 5 Consider a variant of Example 3, in which player a is the only agent capable of free-riding from a two-player coalition, receiving a worth of 9 , as before, when coalition $\{b, c\}$ gets together. However,

$$
v(\{b\},\{\{b\},\{a, c\}\})=v(\{c\},\{\{c\},\{a, b\}\})=0 .
$$

Let us focus for simplicity on a subclass of values $\sigma^{(S, \Pi)}$ that satisfy $(S, \Pi)$-Anonymity, $(S, \Pi)$-Efficiency and $(S, \Pi)$-Monotonicity, for each embedded coalition $(S, \Pi)$, namely those that can be derived through Macho-Stadler et al. (2006, 2007) average approach. Externalities matter only when the three players decide to bargain all together in the grand coalition. Fix $\alpha \in[0,1]$. Then $\sigma^{\alpha}(v)=S h\left(v^{\alpha}\right)$, where $v^{\alpha}$ is the fictitious characteristic function defined as follows: $v^{\alpha}(\{i\})=\alpha v(\{i\},\{\{i\},\{j, k\}\})+(1-\alpha) v(\{i\},\{\{i\},\{j\},\{k\}\})$, $v^{\alpha}(S)=v(S, \Pi)$ for any other $S$. Simple computations lead to the following payoff configuration: $x^{\alpha}(\{N\})=(7.5+3 \alpha, 8-1.5 \alpha, 8.5-1.5 \alpha) ; x^{\alpha}(\{a\},\{b, c\})=(9,7,7)$; $x^{\alpha}(\{b\},\{a, c\})=(6.5,0,6.5) ; x^{\alpha}(\{c\},\{a, b\})=(6,6,0) ; x^{\alpha}(\{a\},\{b\},\{c\})=(0,0,0)$.

The grand coalition is strictly dominant if and only if $\alpha \in] 1 / 2,2 / 3[$. Otherwise, other inefficient coalition structures are likely to form in some circumstances. For two extreme examples, consider $\alpha=0$ or 1 . In the latter case, the coalition $\{b, c\}$ is dominant (not strictly for agent $c$ ). In the former case, the core is the set of partitions $\{N\}$ and $\{\{a\},\{b, c\}\}$. In the sequential game, the equilibrium is the latter partition if player a is the first mover, but it is the grand coalition otherwise.

\subsection{Sufficient Conditions for Efficiency}

If the surplus to share when everyone cooperates is large enough, then one expects the grand coalition to be efficient, and actually to form. We present two results that confirm this intuition. Needless to say, the sufficient conditions to guarantee overall efficiency are rather restrictive, given the robust examples presented in the previous subsection.

Proposition 3 Suppose that the value $\sigma^{(S, \Pi)}$ satisfies $(S, \Pi)$-Anonymity, $(S, \Pi)$-Efficiency and Weak $(S, \Pi)$-Marginality. Consider a partition function that can be decomposed into the sum of a symmetric partition function $u$ and a characteristic function $v$. If

1. $\frac{u(N,\{N\})}{n} \geq \frac{u(S, \Pi)}{s}$, for each embedded coalition $(S, \Pi)$, and

2. $v$ is convex: $v\left(S^{\prime}\right)-v\left(S^{\prime} \backslash\{i\}\right) \geq v(S)-v(S \backslash\{i\})$ for each $i, S$, $S^{\prime}$ such that $i \in S \subsetneq S^{\prime}$, and

3. either all the inequalities appearing in 1, or all the inequalities appearing in 2, are strict, 
then $\{N\}$ is the unique efficient coalition structure, and it is strictly dominant.

Proof: Condition 1 implies that the grand coalition is efficient for $u$. Indeed, let $\Pi=$ $\left(S_{1}, \ldots, S_{K}\right)$ be a partition of $N$. We have:

$$
\sum_{k=1}^{K} u\left(S_{k}, \Pi\right)=\sum_{k=1}^{K} \sum_{i \in S_{k}} \frac{u\left(S_{k}, \Pi\right)}{s_{k}} \leq \sum_{i \in N} \frac{u(N,\{N\})}{n}=u(N,\{N\}) .
$$

It is not difficult to check that convexity implies superadditivity for $v$. Hence the grand coalition is efficient for $v$. Condition 3 then implies that the grand coalition is the only efficient coalition structure for $u+v$.

Proposition 1 implies that

$$
x_{i}(\Pi)=\sigma_{i}^{(S, \Pi)}(u+v)=\frac{u(S, \Pi)}{s}+S h_{i}^{S}\left(v_{S}\right),
$$

for each $i \in S$, and each embedded coalition $(S, \Pi)$ such that $i \in S$.

Observe that

$$
S h_{i}^{S}\left(v_{S}\right)=S h_{i}^{N}\left(v^{\prime}\right),
$$

for each $i \in S$, and each $S \subseteq N$, where $v^{\prime}$ is the characteristic function defined by $v^{\prime}\left(S^{\prime}\right)=v\left(S^{\prime} \cap S\right)$, for each $S^{\prime} \subseteq N$. Though a bit tedious, this property can be checked via the formula of the Shapley value.

The convexity of $v$ implies that

$$
v^{\prime}\left(S^{\prime}\right)-v^{\prime}\left(S^{\prime} \backslash\{i\}\right) \leq v\left(S^{\prime}\right)-v\left(S^{\prime} \backslash\{i\}\right)
$$

for each $S^{\prime} \subseteq N$ that contains $i$. Monotonicity for the Shapley value implies that

$$
S h_{i}^{S}\left(v_{S}\right)=S h_{i}^{N}\left(v^{\prime}\right) \leq S h_{i}^{N}(v) .
$$

The inequality is strict, if $v$ is strictly convex.

Combining equation (2) with conditions 1 and 3 from the statement, we conclude that $\{N\}$ is strictly dominant.

Two particular cases of interest are obtained by taking either $u$ or $v$ as the null partition function. We also remark that condition 3 is important for Proposition 3 to hold. For example, suppose $v(S)=s$ for every $S \subseteq N$. For this characteristic function, every partition is in the core and is supported by a subgame perfect equilibrium of the coalition formation game.

If the partition function cannot be decomposed into the sum of a symmetric partition and a characteristic function, then the bounds of Proposition 2 can prove useful to obtain sufficient conditions for efficiency.

Proposition 4 Suppose that the value $\sigma^{(S, \Pi)}$ satisfies $(S, \Pi)$-Anonymity, $(S, \Pi)$-Efficiency and $(S, \Pi)$-Monotonicity. If the partition function $v$ is such that

$$
\nu_{i}^{(S, \Pi)}(v)<\mu_{i}^{(N,\{N\})}(v),
$$

for each member $i$ of $S$, and each embedded coalition $(S, \Pi)$, then $\{N\}$ is the unique efficient coalition structure, and it is strictly dominant. 
Proof: Let $\Pi=\left(S_{1}, \ldots, S_{K}\right)$ be a partition of $N$. We have:

$$
\begin{aligned}
\sum_{k=1}^{K} v\left(S_{k}, \Pi\right) & =\sum_{k=1}^{K} \sum_{i \in S_{k}} \sigma_{i}^{\left(S_{k}, \Pi\right)}(v) \\
& \leq \sum_{k=1}^{K} \sum_{i \in S_{k}} \nu_{i}^{(S, \Pi)}(v) \\
& <\sum_{i \in N} \mu_{i}^{(N,\{N\})}(v) \\
& \leq \sum_{i \in N} \sigma_{i}^{(N,\{N\})}(v) \\
& =v(N,\{N\}) .
\end{aligned}
$$

Hence the grand coalition is the unique efficient coalition structure. The rest of the proof follows from Proposition 2 and equation 3.

Remember that the grand coalition was not always strictly dominant in Example 5. An inefficient coalition structure was a plausible outcome for some monotonic values. Using the bounds as calculated in de Clippel and Serrano (2008), Proposition 4 implies that in that example the grand coalition is strictly dominant for any monotonic value if $v(N)>28.5$, or if $v(\{b, c\})<9.5$.

\subsection{Related Literature}

The resolution of the second stage of our cooperation process (cf. Subsection 3.1) is inspired by Aumann and Drèze's (1974, Section 3) adaptation of the Shapley value to characteristic functions with an existing coalition structure. They reproduce in Section 12.6 an informal argument due to Michael Maschler to determine endogenously the coalition structure that forms. They observe that the final outcome may be inefficient for some superadditive characteristic functions. Example 4 formalizes and strengthens that observation, showing that an inefficient partition may be strictly dominant. In this context, Proposition 3 offers an interesting sufficient condition for efficiency. Our contribution is also to extend this methodology to problems with externalities. To the best of our knowledge, Shenoy (1979) is the only paper that studies this two-stage cooperation process for characteristic functions. He introduces a dynamic solution to discuss situations where the core of the coalition formation game is empty. He studies the properties of and relations that may exist between - the core and the dynamic solution, for different solutions of the second stage. His analysis of the Shapley value (see his Section 7) focuses mainly on "simple games."

In this paper we followed the idea that players have no option to argue in favor of a larger payoff by appealing to cooperative opportunities with players with whom they decided not to bargain. Of course alternative scenarios may be relevant as well. Along these lines, Hart and Kurz (1983) propose to apply the Owen value once a coalition structure is formed. By definition, outcomes are always efficient in their cooperation process, since coalitions are interpreted as unions that form to get a better share of the total profit. Even though they focus only on characteristic functions, some externalities exist when the coalitions form, as a consequence of the definition of the Owen value. Their paper contains a discussion of the core, based on different possible reactions of the players left behind after the formation of a blocking coalition. 
More recent papers focus on the problem of coalition formation, leaving the second stage of the cooperation process as a black box. Each player derives some utility from his membership to the different coalitions. Bogomolnaia and Jackson (2002) study the existence of core stable partitions and other weaker notions of stability. Banerjee et al. (2001) introduce the concept of top coalition (similar to our concept of strict dominance when there are no externalities) to prove the non-emptiness of the core in some economic applications; see also Alcalde and Revilla (2004). Proposition 3, applied to the case $u=0$, could be added to that list of applications that admit a strictly dominant partition.

The problem of coalition formation for partition functions, or more generally for games with strategic externalities, has been the subject of a growing literature over the past few years; see Bloch (2002). Bloch (1996) for instance studies a specific bargaining procedure to solve the first stage of the cooperation process, while the second stage is solved by a fixed arbitrary division rule (hence adding the possibility of externalities to the hedonic games). Many other authors focus on specific bargaining procedures to simultaneously determine the coalitions that form and the distribution of the surplus (Ray and Vohra (1999), Montero (1999), and of course Maskin (2003)). Ray and Vohra (2001) study a core-like solution, assuming that only subcoalitions can block. Our observation that inefficiency may be due entirely to the presence of externalities (see Example 3) confirms similar conclusions previously obtained in these different frameworks. Given the complexity of some of these models, attention is often restricted to symmetric partition functions. Proposition 3 is a result of that type when $v$ equals 0 .

It is important to emphasize though that all these results on the efficiency of the "equilibrium" outcomes strongly depend on the type of cooperation process one considers. One can construct cooperation processes that lead to an efficient outcome with or without externalities, and one can construct other bargaining processes that lead to inefficient outcomes only in the presence of externalities. In the end, the interest of the different results should be judged on their robustness and on the appeal of the cooperation process under study. In scenarios in which players recognize that it may be in their best interest to restrict the set of players with whom to negotiate when there is an ex-ante commitment to a known distribution rule, robust conclusions were sought here.

\section{References}

Alcalde, J. and P. Revilla (2004), "Researching with Whom? Stability and Manipulation," Journal of Mathematical Economics 40, 869-887.

Aumann, R. J. and J. Drèze (1974), "Cooperative Games with Coalition Structures," International Journal of Game Theory 3, 217-237.

Banerjee, S., H. Konishi and T. Sönmez (2001), "Core in a Simple Coalition Formation Game," Social Choice and Welfare 18, 135-153.

Bloch, F. (1996), "Sequential Formation of Coalitions in Games with Externalities and Fixed Payoff Division," Games and Economic Behavior 14, 90-123.

Bloch, F. (2002), "Coalitions and Networks in Industrial Organization," Manchester School 70, 36-55. 
Bogomolnaia, A. and M. O. Jackson (2002), "The Stability of Hedonic Coalition Structures," Games and Economic Behavior 38, 201-230.

de Clippel, G. and R. Serrano (2008), "Marginal Contributions and Externalities in the Value," Econometrica, forthcoming.

Hafalir, I. E. (2007), "Efficiency in Coalition Games with Externalities," Games and Economic Behavior 61, 242-258.

Hart, S. and M. Kurz (1983), "Endogenous Formation of Coalitions," Econometrica 51, 1047-1064.

Hart, S. and A. Mas-Colell (1996), "Bargaining and Value," Econometrica 64, 357-380.

Macho-Stadler, I., D. Pérez-Castrillo and D. Wettstein (2006), "Efficient Bidding with Externalities," Games and Economic Behavior 57, 304-320.

Macho-Stadler, I., D. Pérez-Castrillo and D. Wettstein (2007), "Sharing the Surplus: An Extension of the Shapley Value for Environments with Externalities," Journal of Economic Theory 135, 339-356.

Maskin, E. (2003), "Bargaining, Coalitions and Externalities," Presidential Address to the Econometric Society, Institute for Advanced Study, Princeton.

Montero, M. (1999), "Coalition Formation in Games with Externalities," CentER Discussion Paper 121, University of Tilburg.

Pérez-Castrillo, D. and D. Wettstein (2001), "Bidding for the Surplus: A Non-Cooperative Approach to the Shapley Value," Journal of Economic Theory 100, 274-294.

Ray, D. and R. Vohra (1999), "A Theory of Endogenous Coalition Structures," Games and Economic Behavior 26, 286-336.

Ray, D. and R. Vohra (2001), "Coalitional Power and Public Goods," Journal of Political Economy 109, 1355-1384.

Shenoy, P. P. (1979), "On Coalition Formation: a Game-Theoretical Approach," International Journal of Game Theory 8, 133-164.

Thrall, R. M. and W. F. Lucas (1963), "n-Person Games in Partition Function Form," Naval Research Logistics Quarterly 10, 281-298. 